\title{
FURTHER OBSERVATIONS ON THE PATHOLOGY OF PULMONARY EMPHYSEMA IN CHRONIC BRONCHITICS
}

\author{
BY \\ BRIAN E. HEARD \\ From the Department of Morbid Anatomy, Postgraduate Medical School of London
}

(RECEIVED FOR PUBLICATION JULY 28, 1958)

In a previous communication, methods for demonstrating the pathology of pulmonary emphysema were described (Heard, 1958). Centrilobular and diffuse varieties were illustrated, each being localized or widespread.

The present communication records the detailed appearances of normal lungs by these methods and compares them with the changes of diffuse emphysema.

\section{MethodS}

Full details of the methods for pressure-fixing lungs and impregnating slices with barium sulphate were given previously. Each lung was injected through the main bronchus with $20 \%$ formalin at a pressure of 10 to 12 in. $(25-30 \mathrm{~cm}$.) and the pressure was maintained for a minimum period of three days by an apparatus described previously (Heard, 1958). All air spaces were evenly and gently distended and the lung did not shrink or distort during fixation. Whole slices of lung were cut with a ham knife supported on rails $0.8 \mathrm{~cm}$. high. A selected slice was soaked consecutively in aqueous solutions of barium nitrate and sodium sulphate so that the semitranslucent respiratory tissue became delicately whitened with precipitated barium sulphate. It was photographed in water.

The photographs of whole slices have an accompanying scale, while the detailed surface photographs have been enlarged to a standard magnification of 20 times.

\section{Findings}

Appearances of Normal Lungs.-In order to recognize the mildest forms of diffuse emphysema it is necessary to know the appearances of normal lungs by these methods. Since the sizes of the air spaces tend to increase with age, the appearances at three ages will be described.

The first lung is from a girl of $6 \frac{1}{2}$ years. A sagittal slice of the left lung is shown diagrammatically in Fig. 1a. The upper and lower lobes are similar in size (as in the adult), but a special feature is prominent loose connective tissue between secondary lobules, especially in the lingular and anterior basal segments, causing well-defined subdivisions in the lung. The normal lung slice at any age has a characteristic consistency in water. It feels soft to the fingertips, but yields slowly to compression, due to the maze of intact respiratory channels.

Fig. 2 is a surface photograph from the apical segment of the lower lobe magnified 20 times. In the right upper quadrant of the photograph is a terminal bronchiole with accompanying artery. It lies near the centre of a secondary lobule, the periphery of which is demarcated to the left by a septum (with veins). The terminal bronchiole gives rise to a respiratory bronchiole in which the openings of alveoli can be made out. Further orders of respiratory bronchioles are cut in varying planes from there outwards to the septum. Two sizes of air spaces are apparent in this maze. The largest are respiratory bronchioles which are about $0.2 \mathrm{~mm}$. diameter. The smallest are alveoli scattered along the walls of the respiratory bronchioles and measuring approximately $0.1 \mathrm{~mm}$. diameter.

Fig. 1b shows the normal left lung from a man of 50. The upper and lower lobes are again similar in size. Interlobular septa could be seen as white lines in the centre of the apical segment and in the anterior segment of the upper lobe. In the lingula and in the centre of the lower part of the lower lobe the septa were thickened by connective tissue. This was a persistence of the lobular separation which was more obvious in the child (Fig. 1a). The dust pigment in the normal adult lung accumulates predominantly in the upper two-thirds of the upper lobe and in the upper and anterior parts of the lower lobe. It is usually least in the lingula (or middle lobe) and in the posterior basal segment. The pigment collects near the centres of the secondary lobules, and hence shows a distinct tendency to avoid the fibrous septa. Where septa are scanty or absent the pigment is a useful guide to lobular structure. 

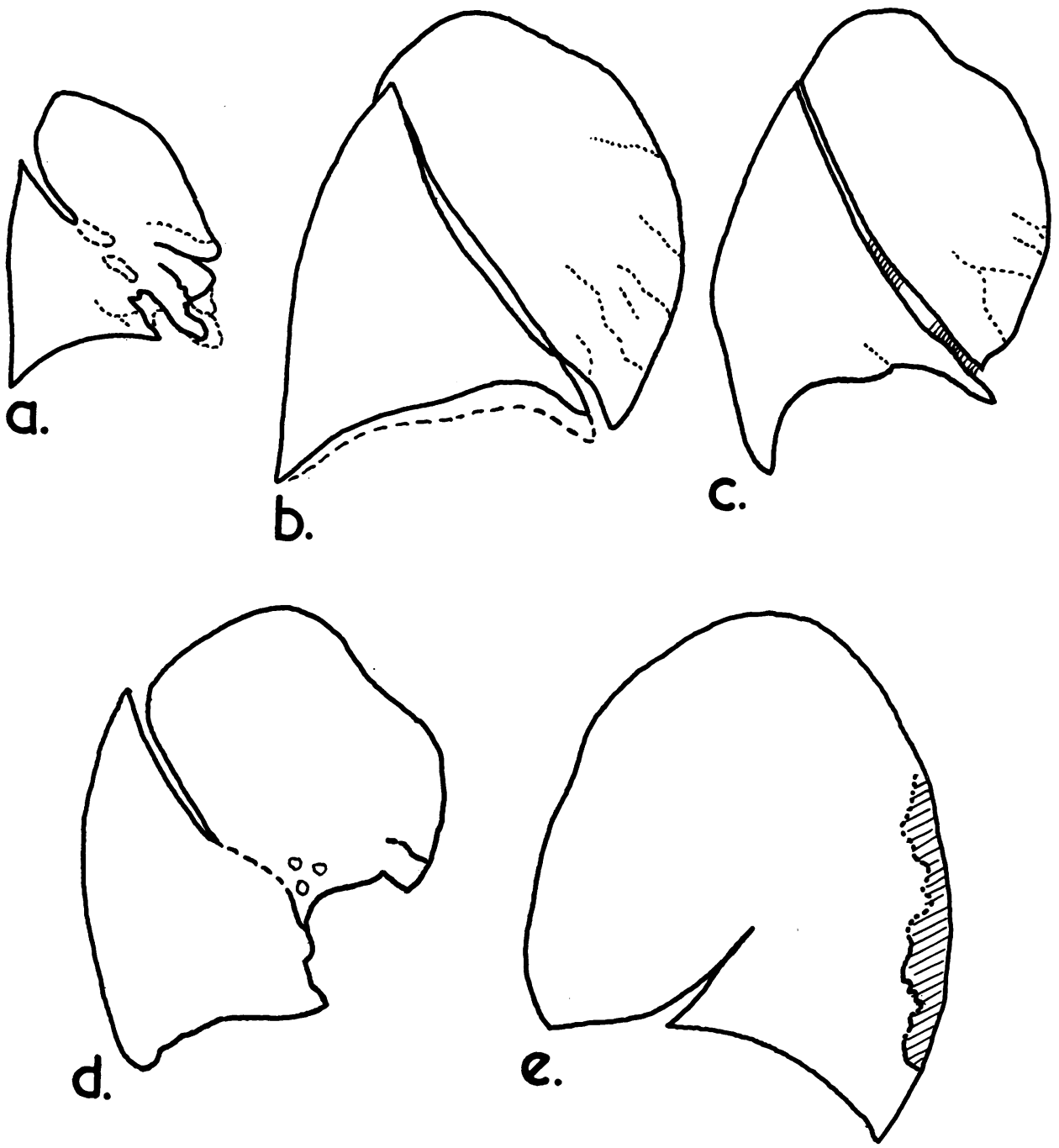

FIG. 1.-Diagrams to show some gross features of lung slices. (a) Girl of $6 \frac{1}{2}$ years, normal lung; r(b) man of 50 years, normal lung; (c) man of 69 years, normal lung; (d) woman of 69 years, shrunken bronchiectatic lingula, mild diffuse emphysema of most of upper lobe; (e) man of 71 years, moderate diffuse emphysema, and superficial emphysema back of lower lobe $(\times 0.25)$.

Fig. 3 shows details of the centre of the lingula. In the right upper quadrant of the photograph is a terminal bronchiole. The edge of the secondary lobule is in the left lower quadrant. In the main mass of the lobule, between these landmarks, lies an intricate maze of succeeding orders of respiratory bronchioles bearing alveoli. As in the child's lung, large and small air spaces can be made out quite clearly. The respiratory bronchioles are between 0.2 and $0.4 \mathrm{~mm}$. in diameter. The alveoli are 0.1 to $0.15 \mathrm{~mm}$. diameter, with an occasional $0.2 \mathrm{~mm}$. maximum. In Fig. 4 the anterior basal segment is pictured. Most of the respiratory bronchioles are about $0.3 \mathrm{~mm}$. diameter. To the right is a prominent lymphatic lying in an interlobular septum.

The third normal example is the left lung from a man of 69 (Fig. 1c). The lobes are again similar in size. Pale foci of terminal bronchopneumonia picked out the centres of the secondary lobules of the posterior basal segment and of the lower part of the apical segment of the lower lobe. The distribution of these 
FIG. 2-Details of the apical segment of the lower lobe of Fig, 1a showing a terminal bronchiole (in the right upper quadrant) giving off respiratory bronchiole with a few alveoli in the wall. Successive orders of respiratory bronchioles, lined by alveoli, lead across the lobule the bordering septum on the left $(\times 20)$.

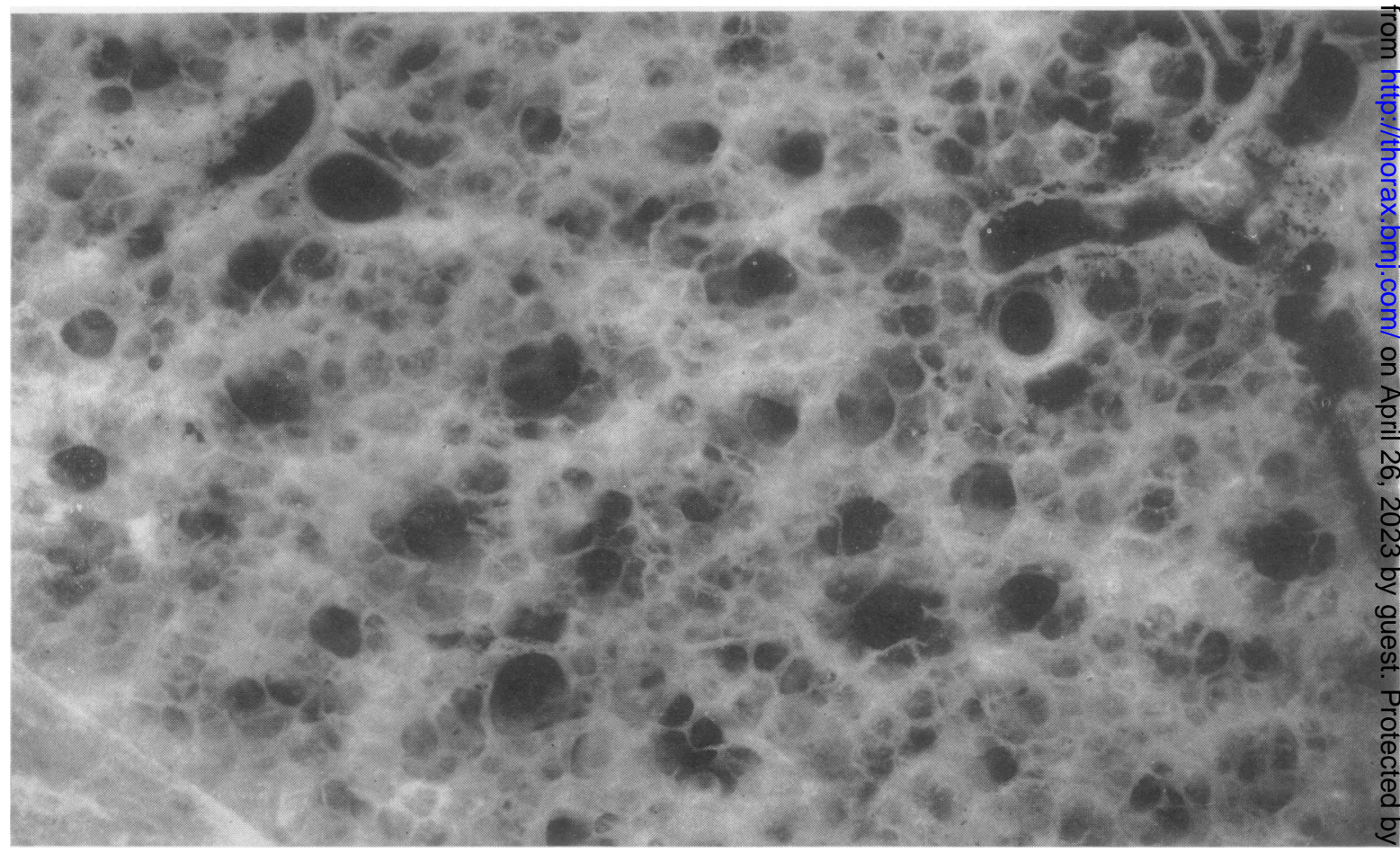

FIG. 3.-Details of the lingula of the normal lung in Fig. 1b. Between the terminal bronchiole (in the right upper corner) and the $s$ eptum (in tie left lower corner) lies an intricate maze of respiratory bronchioles (large spaces) and alveoli (smallest spaces) $(\times 20)$. 


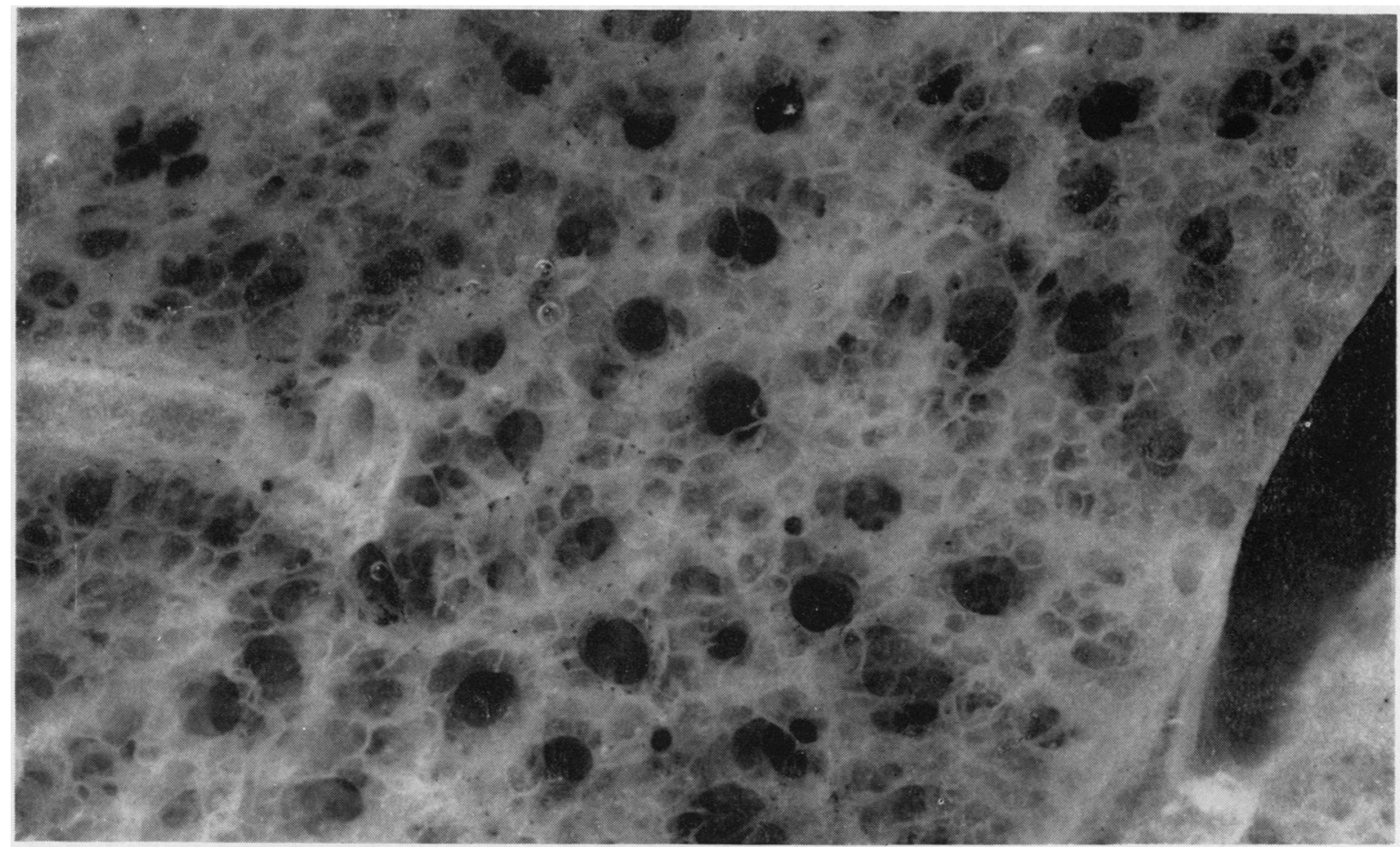

F1G. 4. -Details of the anterior basal segment of Fig. 1b. The large spaces are respiratory bronchioles and the smallest are alveoli ( $\times 20)$.

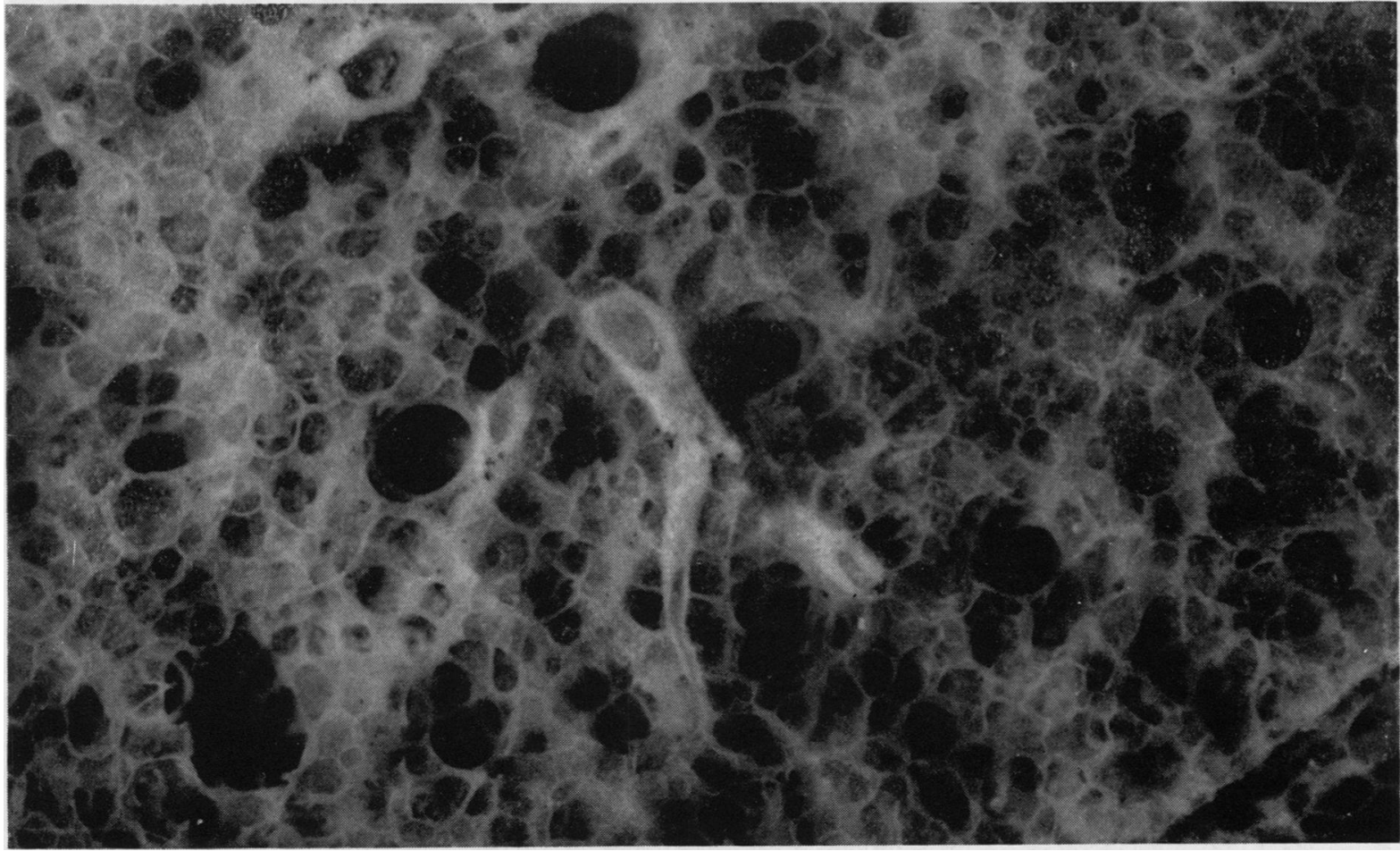

Fig. 5.-Details of the middle of the upper lobe of Fig. 1c. Respiratory bronchioles (large holes) and alvooli (small holes) are easily identified ( $\times$ 20). 
inflammatory foci was similar within the lobule (not in the lung as a whole) to centrilobular pigment and to centrilobular emphysema. It is possible, as has been suggested by several authors, that a bronchiolitis similar to this is responsible for the characteristic localization of centrilobular emp'ysema. Septa were very distinct in this lung, especially in the upper lobe. The septa in the lingula had the same abundant loose connective tissue as the previous examples. Centrilobular dust pigment was scanty and mostly confined to the upper third of the upper lobe with a little in the apical segment of the lower lobe. The finer detail of the middle of the left upper lobe is shown in Fig. 5. The respiratory bronchioles are slightly larger than in either of the previous specimens, ranging from 0.3 to $0.5 \mathrm{~mm}$. diameter but rarely more than this. The alveoli are also slightly larger than previously, measuring 0.15 to $0.2 \mathrm{~mm}$. diameter.

To conclude this description of normal lungs it should be pointed out that Professor Jethro Gough (1957) was able to estimate correctly the age of the third lung by noting the furthest distance from the eyes that large air spaces could be seen. The largest spaces are respiratory bronchioles. Those of the girl's lung could be seen in the barium-impregnated slice up to a distance of 15 in. $(37.5 \mathrm{~cm}$.), which is elbow length approximately from the eyes. Those of the man of 50 years could be seen up to $25 \mathrm{in}$. $(62.5 \mathrm{~cm}$.) from the eyes, which is at arm's length. Those of a man of 69 years illustrated in Fig. 5 could be seen up to a distance of 35 to 40 in. (87.5 to $100 \mathrm{~cm}$.), which is a foot beyond arm's length.

Mild Diffuse EMPhysema.-From the present work, it seems that the mildest grade of diffuse emphysema may be described as a dilatation of fine respiratory tissue beyond the normal range for that age. In more obvious forms of diffuse emphysema there is atrophy of the walls of fine air spaces. In either of these states any of the fine structures beyond the terminal bronchioles may be affected. Centrilobular emphysema is a distinct pattern and grouped separately.

Fig. 1d is the left lung of a woman of 69 who died of cor pulmonale. The lingula is shrunken and bronchiectatic. Other slices showed bronchiectasis in the anterior basal segment. In the apical segment of the lower lobe there was some patchy pigmented centrilobular emphysema. The point of present interest, however, is the diffuse emphysema of the upper lobe. Respiratory bronchioles could be seen to a distance of twice arm's length with the naked eye. Fig. 6 shows

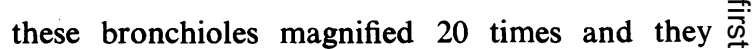
may reach $0.65 \mathrm{~mm}$. diameter and sometimes more. Most alveoli are about $0.2 \mathrm{~mm}$. diameter, but occasionally reach $0.25 \mathrm{~mm}$. or $0.3 \mathrm{~mm}$. Their measured size is not as striking as would be expected from general appearances, but a clear idea of the degree of dilatation is achieved by comparing this slice with a normal slice of a similar age side by side under the dissecting microscope.

This case illustrates early diffuse emphysema in $\vec{x}$ which the diameters of the largest respiratory bronchioles and alveoli are greater than the normal for that age. Shrinkage of the bronchi- in ectatic segments may be responsible for this by leading to compensatory emphysema of the upper lobe.

Moderate Diffuse Emphysema.-Many cases of diffuse emphysema are a grade more severe. The affected parts of a slice of lung are easily compressed by the finger-tips, and when water is drained from the slice the emphysematous tissue collapses and the surface sinks below the level of normal lung. Without barium sulphate impregnation it has a gelatinous appearance $\stackrel{2}{\varrho}$ as it floats in water. With barium sulphate $\mathbb{Q}$ impregnation the changes in fine detail become $\overrightarrow{\vec{A}}$ clear, especially when viewed by the dissecting microscope.

Fig. 1e is the right lung of a man of 71 who had chronic bronchitis for some years. The lung is larger than normal; oblique and transverse fissures are incomplete (probably a congenital $\underset{x}{x}$ defect); the interlobular septa were distinct in the $\frac{0}{3}$ anterior one-third of the lung. Examination of the specimen with the dissecting microscope 0 revealed a fine change illustrated in Fig. $7, ₹$ especially in the posterior half of the upper lobe. $\frac{\text { ? }}{5}$ Ir comparison with the normal lung of a similar $>$ age (Fig. 5) parts of this specimen show loss of substance. The normal distinct pattern of N respiratory bronchioles and alveoli is replaced by a less regular pattern of very thin air space walls $\tilde{O}$ through which blood vessels are now clearlyc visible.

Also shown in Fig. 1e is a narrow zone ofe superficial emphysema in the lower lobe $\mathbb{D}$ posteriorly. Its greatest depth is $2 \mathrm{~cm}$., and it ${ }^{+}$ ceases about $5 \mathrm{~cm}$. above the inferior border. Milder grades of this condition have been seen on $\frac{\mathrm{D}}{\mathbb{\Phi}}$ several occasions, but the present example is severe enough to deserve a photograph at a higher $\stackrel{\mathbb{2}}{2}$ magnification (Fig. 8). The normal structure is replaced by a coarse network of pulmonaryo 


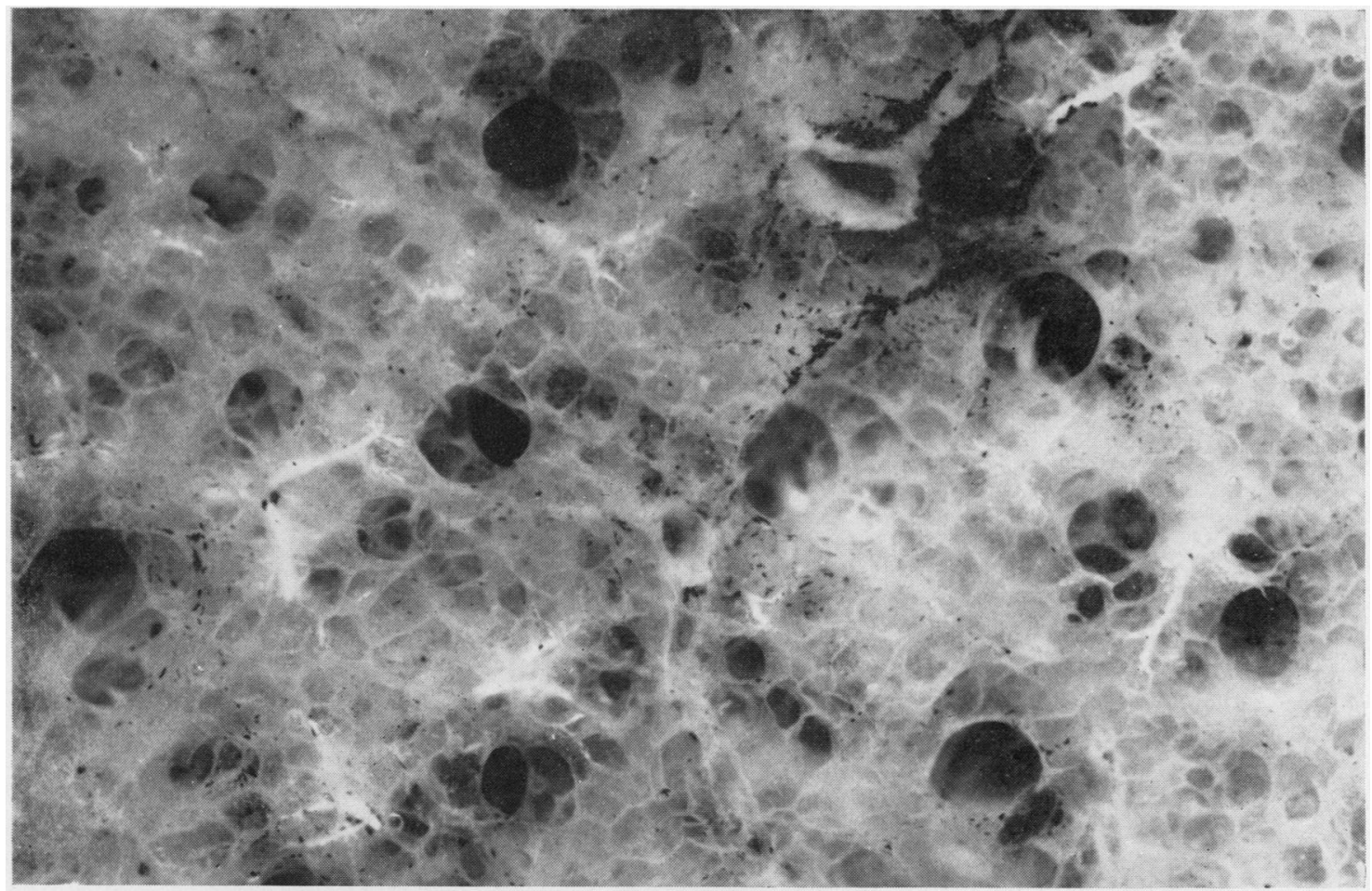

Fig. 6.-Details of the upper lobe of Fig. 1d showing mild diffuse emphysema. The largest respiratory bronchioles and alveoli exceed the usual maximum size $(\times 20)$.

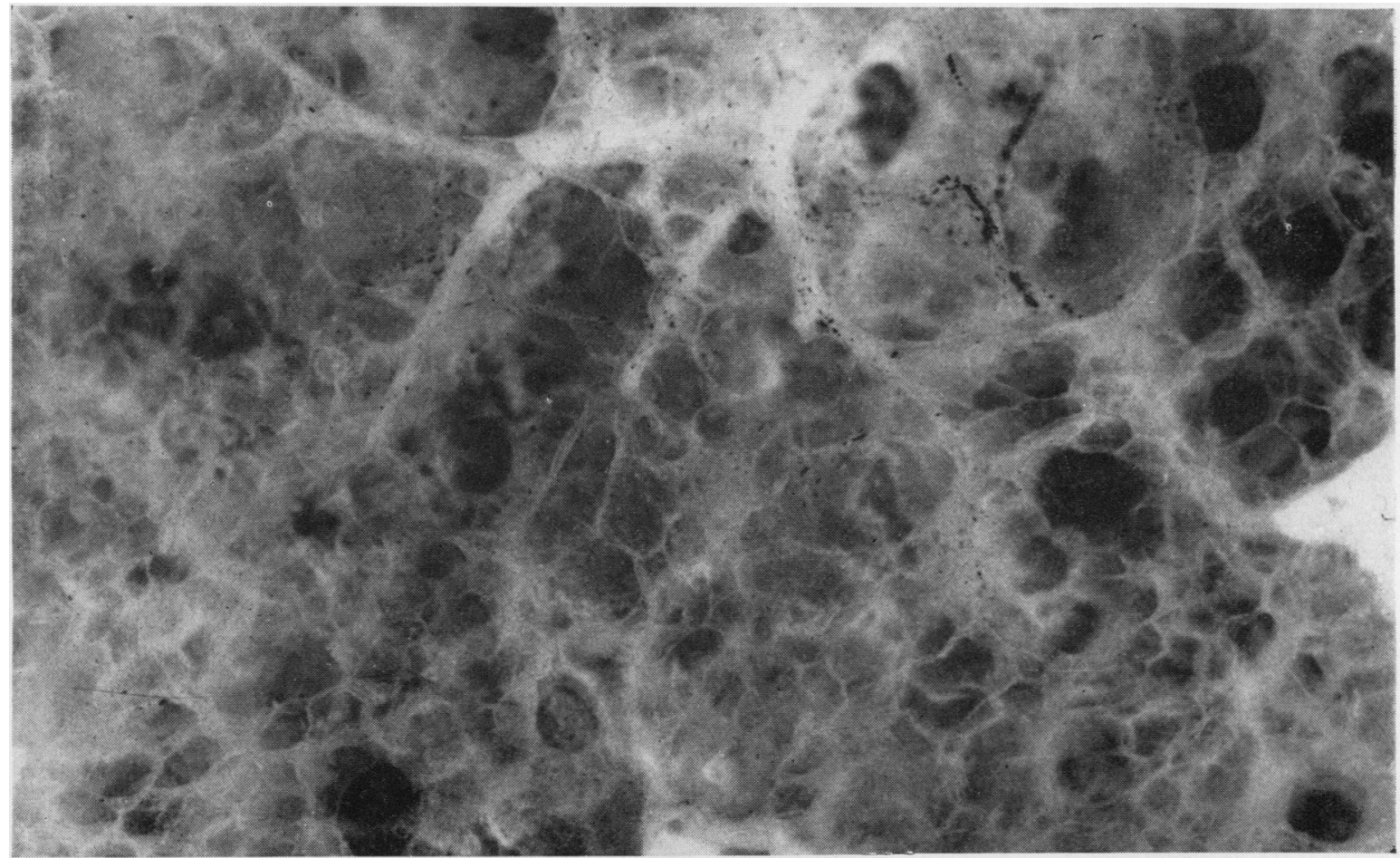

FIG. 7.-Details of the posterior part of the upper lobe in Fig. 1e. Diffuse emphysema is indicated by the thinning of respiratory tissue (leaving a clearer view of vessels) and by the loss of much alveolar detail. This contrasts sharply with the normal lung in Fig. $5(\times 20)$. 


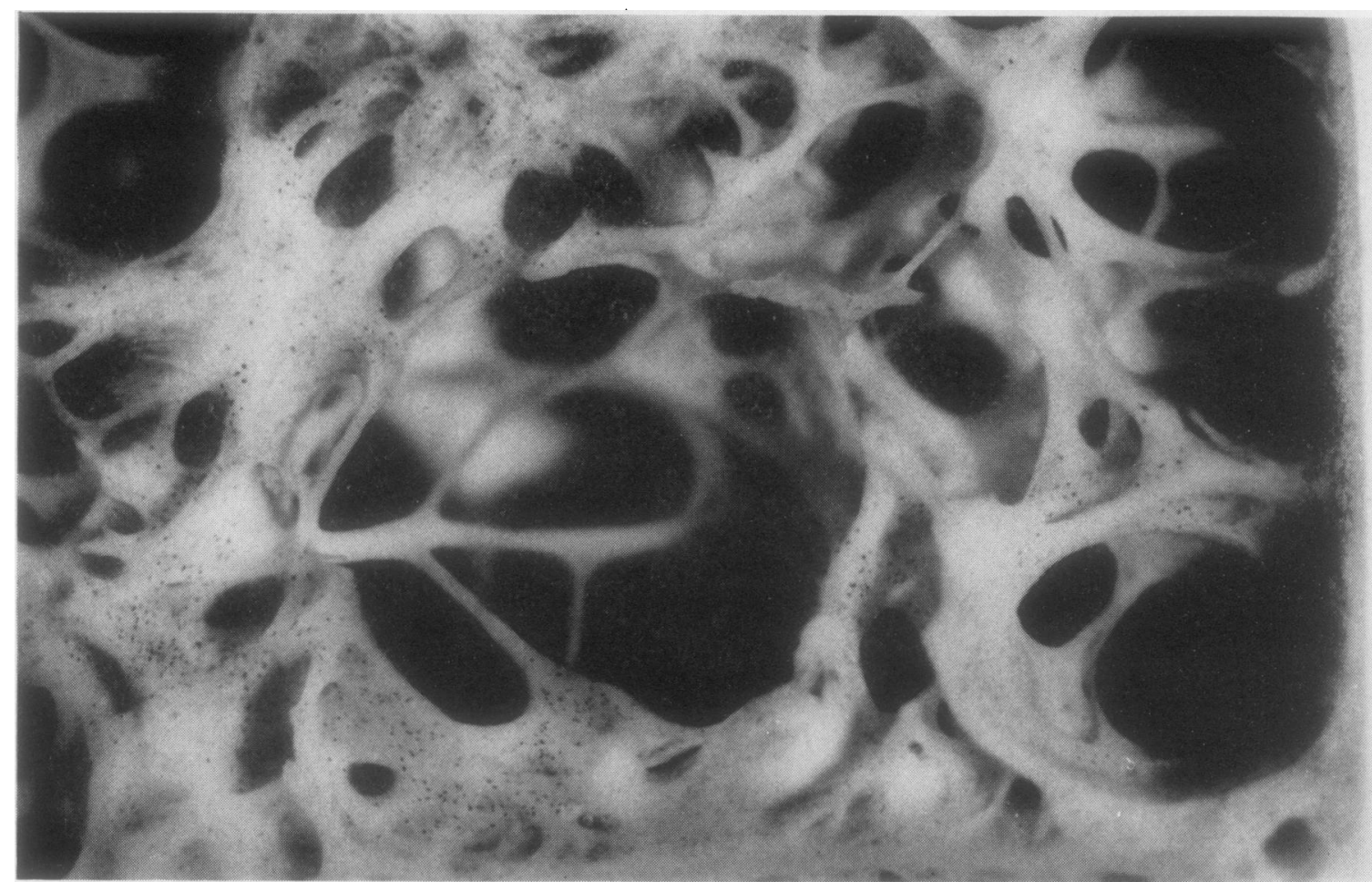

Fig. 8. -Details of the superficial emphysema at the back of the lower lobe in Fig. le. Most of the strands are pulmonary arteries surrounded by fibrous tissue $(\times 20)$.

arteries with fibrosed remnants of respiratory tissue about them. Some of the spaces are $3 \mathrm{~mm}$. in diameter or more. There is a very fine sprinkling of dust pigment over most structures. It remains to be seen whether this subpleural change is related to the widespread diffuse emphysema or is an independent condition. It is best termed "superficial diffuse emphysema" at the moment. Milder grades in this position are common.

Severe Diffuse Emphysema.-An example of severe diffuse emphysema was illustrated in the previous communication (Heard, 1958).

Localized Diffuse EmPhysema.-This variety was also described in the previous publication.

Mixed Diffuse and Centrilobular EMPHYSEMA. - Widespread diffuse emphysema may be combined with centrilobular emphysema. The double picture is illustrated in Fig. 9, which is the left lung of a man of 56 with a history of chronic bronchitis for at least six years. The lower lobe and lower lingular segments are somewhat shrunken and show patchy fibrosis. The rest of the upper lobe and upper half of the lower lobe show advanced widespread diffuse emphysema which is associated with enlargement of the affected parts of the lung. The dark foci of centrilobular emphysema also appear larger than usual, as though they preceded the diffuse change, and had been stretched as the lung enlarged.

Details of part of the anterior segment of the upper lobe are shown in Fig. 10. There is pigmented centrilobular emphysema in the right upper quadrant of the photograph, while the peripheral septum and an included vein are shown in the left lower quadrant. There is diffuse emphysema of the intermediate and peripheral zones of the lobule. In the intermediate zone the changes are severe and bare pulmonary arteries cross large irregular air spaces. No alveoli can be seen and remnants of respiratory bronchioles form vela between the vessels. In the peripheral zone of this lobule the changes are milder.

It has been less usual, to date, to find this degree of diffuse emphysema with centrilobular emphysema. A more common pattern is that shown in Fig. 11, which is the right lung of a man of 56 years who had a chronic cough with 


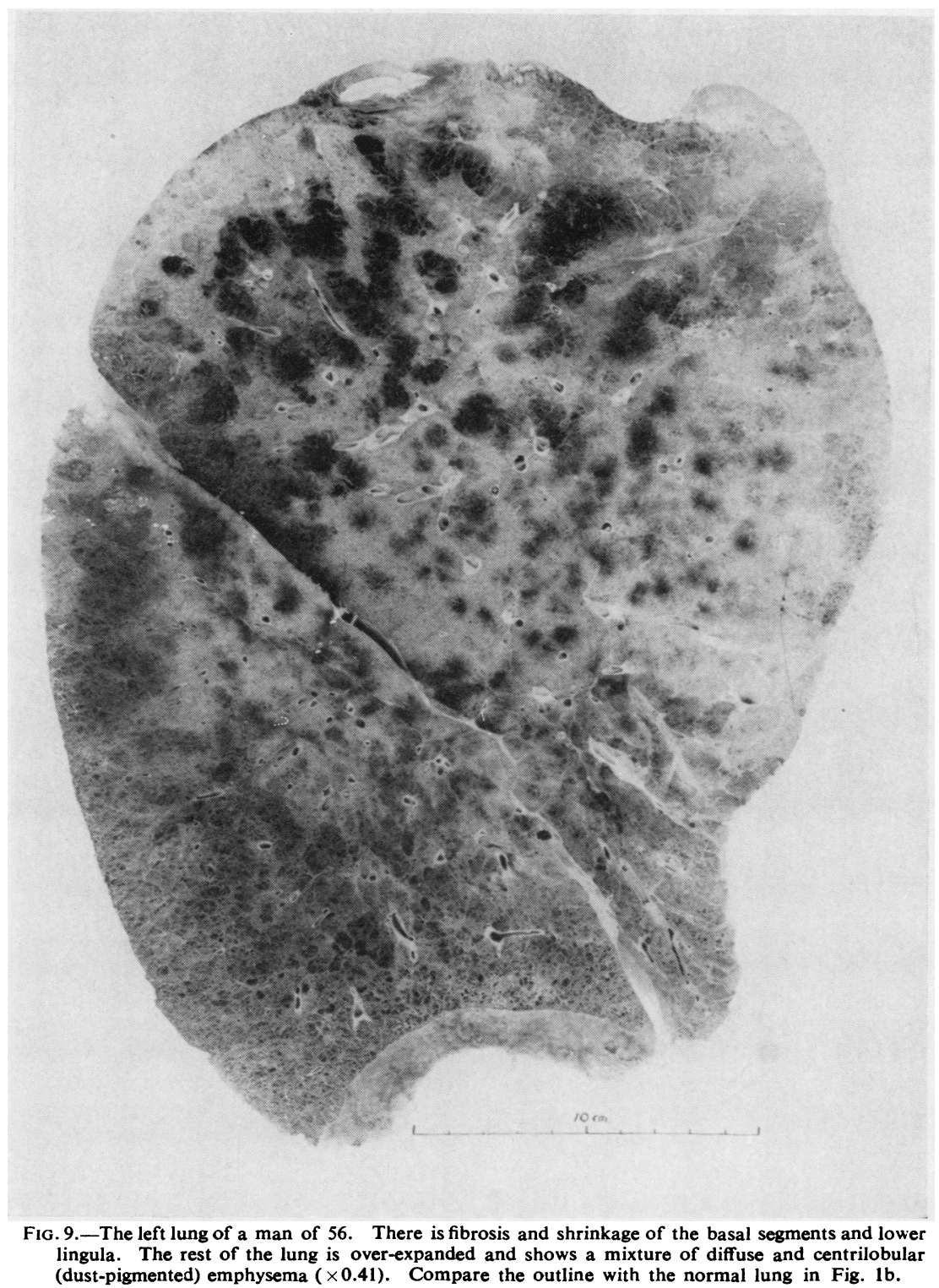

$3 \mathrm{oz}$. (90 ml.) of yellow sputum each morning for some time. He died of renal disease. There is moderately advanced, partly confluent, centrilobular emphysema of much of the upper lobe and a less severe grade in the upper parts of the middle and lower lobes. The upper lobe of this lung is less expanded than that of the previous example, and Fig. 12 shows that the fine respiratory tissue in the peripheral lobular substance has lost only a little of its normal dense pattern. This is a fairly mild grade of diffuse emphysema with some deficiency of alveoli and a general thinning of the respiratory tissue.

A special variety of peripheral lobular diffuse change with centrilobular emphysema, termed " paraseptal emphysema," was illustrated in the previous communication. Fig. 13 is the fine detail from that same case of chronic bronchitis. There is pigmented centrilobular emphysema on the left, and on the right, at the edge of the lobule, is a thickened septum which appears to have been stretched away from the lobular substance in the 


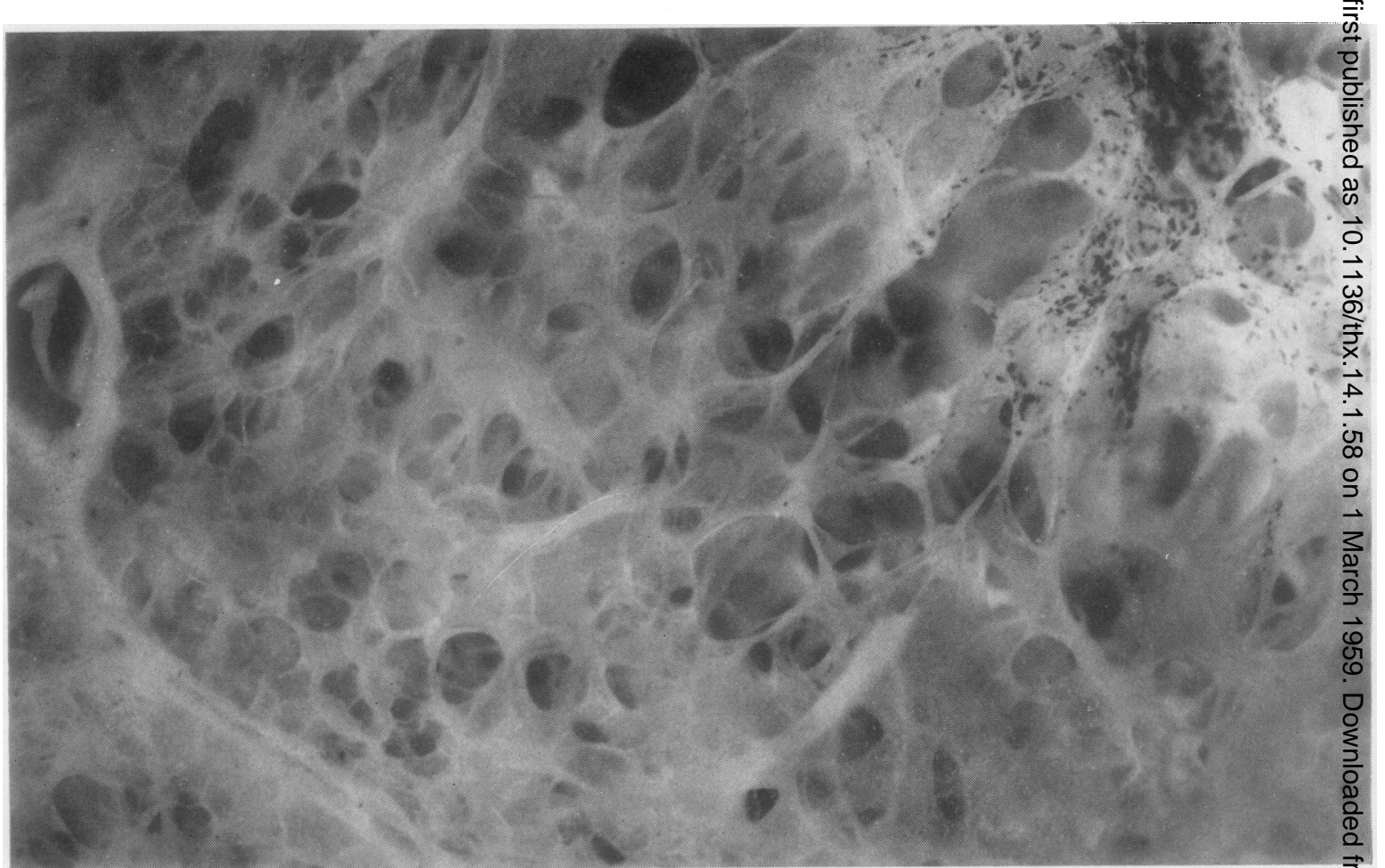

FIG. 10.-Details of the upper lobe of Fig. 9. The centrilobular pigment is in the right upper corner and the peripheral septum lies in to left lower corner. The lung between shows diffuse emphysema with loss of alveoli and thin webs of respiratory tissue stretched between pulmonary arteries $(\times 20)$.

middle of the field. The photograph shows quite clearly that there is a stringy fibrous change in the intermediate zone. The lobule is very severely damaged.

In brief, special methods for displaying emphysema in lung slices as previously described (Heard, 1958) show the fine anatomy of normal lungs to great advantage and allow the detection of the mildest grade of diffuse emphysema with the dissecting microscope.

\section{Discussion}

The provisional classification of emphysema into centrilobular and diffuse types is proving of great value in assessing the pulmonary changes in chronic bronchitis. At the same time, however, it is becoming clear that these types may occur combined and in a multiplicity of patterns.

Centrilobular emphysema is usually quickly identified by the dust pigment. The common distribution is that in Fig. 11 involving the upper part of each lobe and being most intense in the upper lobe. Less commonly a lower lobe may be the principal site. Centrilobular emphysema is being graded as follows: (1) Mild, where lesions are compact and each separated by unpigmented lung; (2) moderate, where the lesions are $\underset{\times}{\stackrel{2}{\jmath}}$ becoming confluent in places; and (3) severe, $\dot{\sigma}$ where confluent lesions involve a large proportion of the lung substance. The extent of the disease $\delta$ is recorded separately on a segmental basis.

Diffuse emphysema is less readily recognized 0 but nevertheless is characteristic in appearance. Pressure fixation and barium impregnation allow the sizes of fine structures to be assessed reliably. In mild degrees of diffuse emphysema the enlarged $O$ respiratory bronchioles become visible to the $N$ naked eye at greater distances than normally. N The enlargement can be confirmed by $O$ measurements with a dissecting microscope (and a graduated eyepiece). In more severe forms the affected parts of the lung slice are easily $\stackrel{\infty}{?}$ compressed by the finger-tips, and collapse when $\square$ water is drained away. The dissecting microscope $\overrightarrow{0}$ shows a general thinning of fine respiratory tissue $\mathbb{\mathbb { D }}$ in any part of affected secondary lobules.

It is possible that some investigators may prefer not to include mild dilatation of air spaces without destruction under the term diffuse emphysema, but 0 


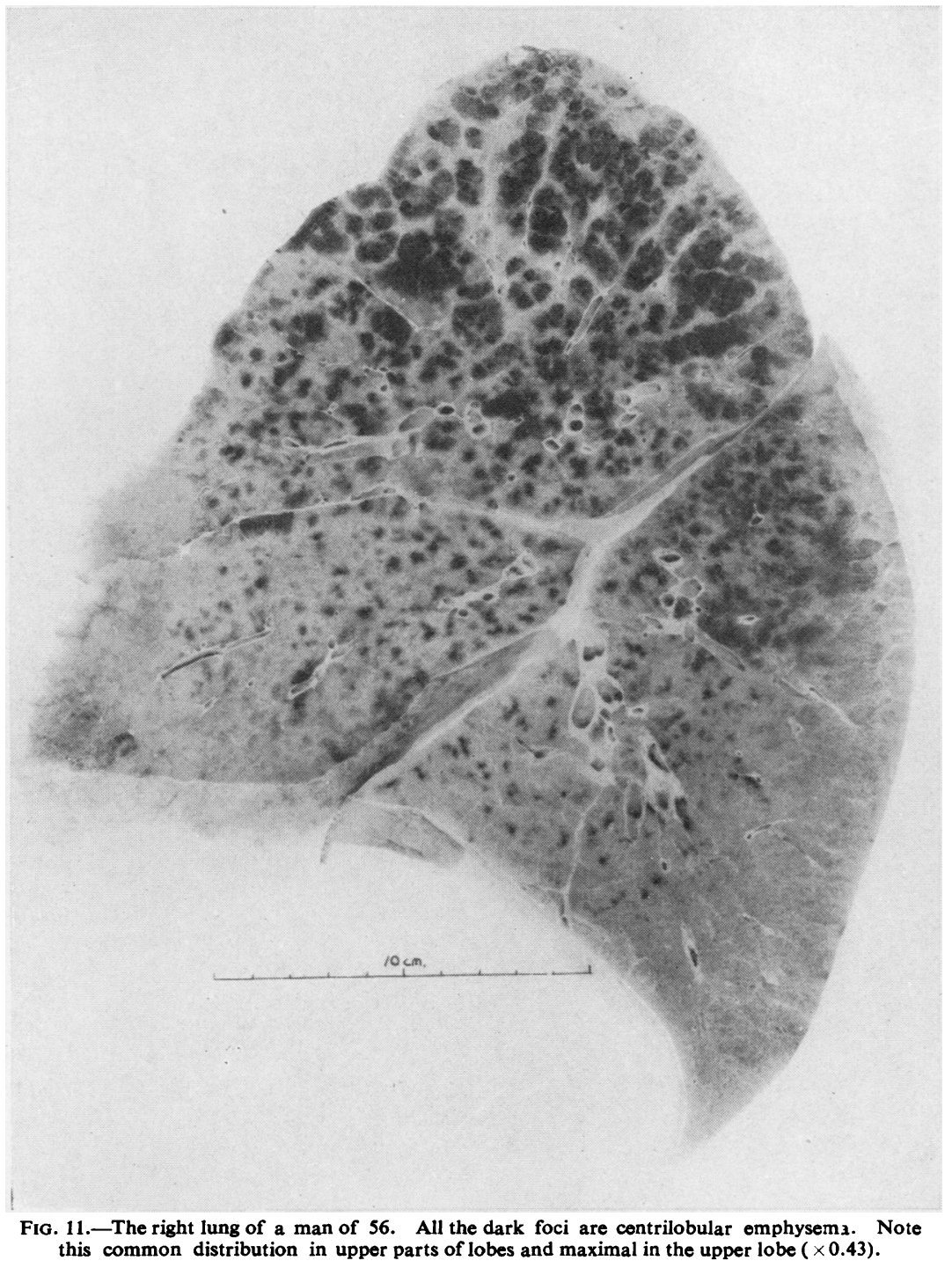

it is included here because dilatation is evident in lungs affected by more obvious grades of diffuse emphysema and may be part of the mechanism of their development (e.g., Figs. 1e and 9).

Measurements of the air spaces were made with the graduated eyepiece of the dissecting microscope or with a ruler on photographs of known magnification. The high-power illustrations are all magnified 20 times $(2 \mathrm{~mm}$. in photograph $=$ $0.1 \mathrm{~mm}$. of lung). Normal respiratory bronchioles ranged from $0.2 \mathrm{~mm}$. in the girl of $6 \frac{1}{2}$ years up to a maximum of $0.5 \mathrm{~mm}$. in the man of 69 years. Normal alveoli ranged from $0.1 \mathrm{~mm}$. in the girl to a maximum of $0.2 \mathrm{~mm}$. in the man of 69 years. Gough's method of estimating quickly the size of air spaces by noting the furthest distance from the naked eye that they can be seen has been mentioned. Leopold and Gough (1957) said that in generalized emphysema the air spaces were individually identifiable even when the section was held at arm's length. In the present preparations the earliest grade of diffuse emphysema was seen at twice arm's length. Hartroft and Macklin (1944) used several schemes for measuring the diameter of alveoli, and arrived at an average of about $0.19 \mathrm{~mm}$. in a woman of 31 years. The 


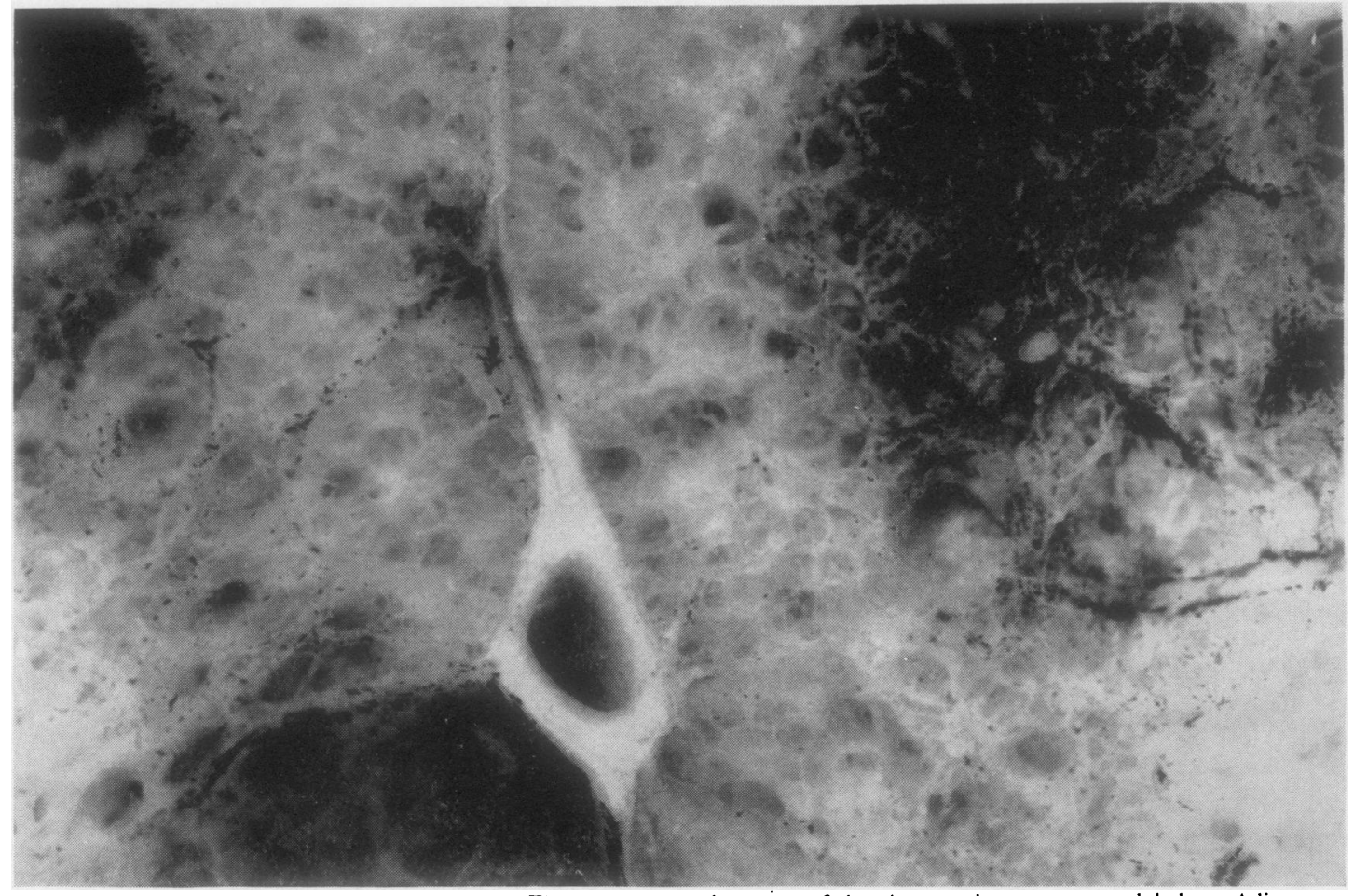

Fig. 12.-Details of the upper lobe of Fig. 11. The septum near the centre of the photograph separates two lobules. Adjacent lung (white) shows slight loss of alveolar detail. Centrilobular emphysema (pigmented) is present $(\times 20)$.

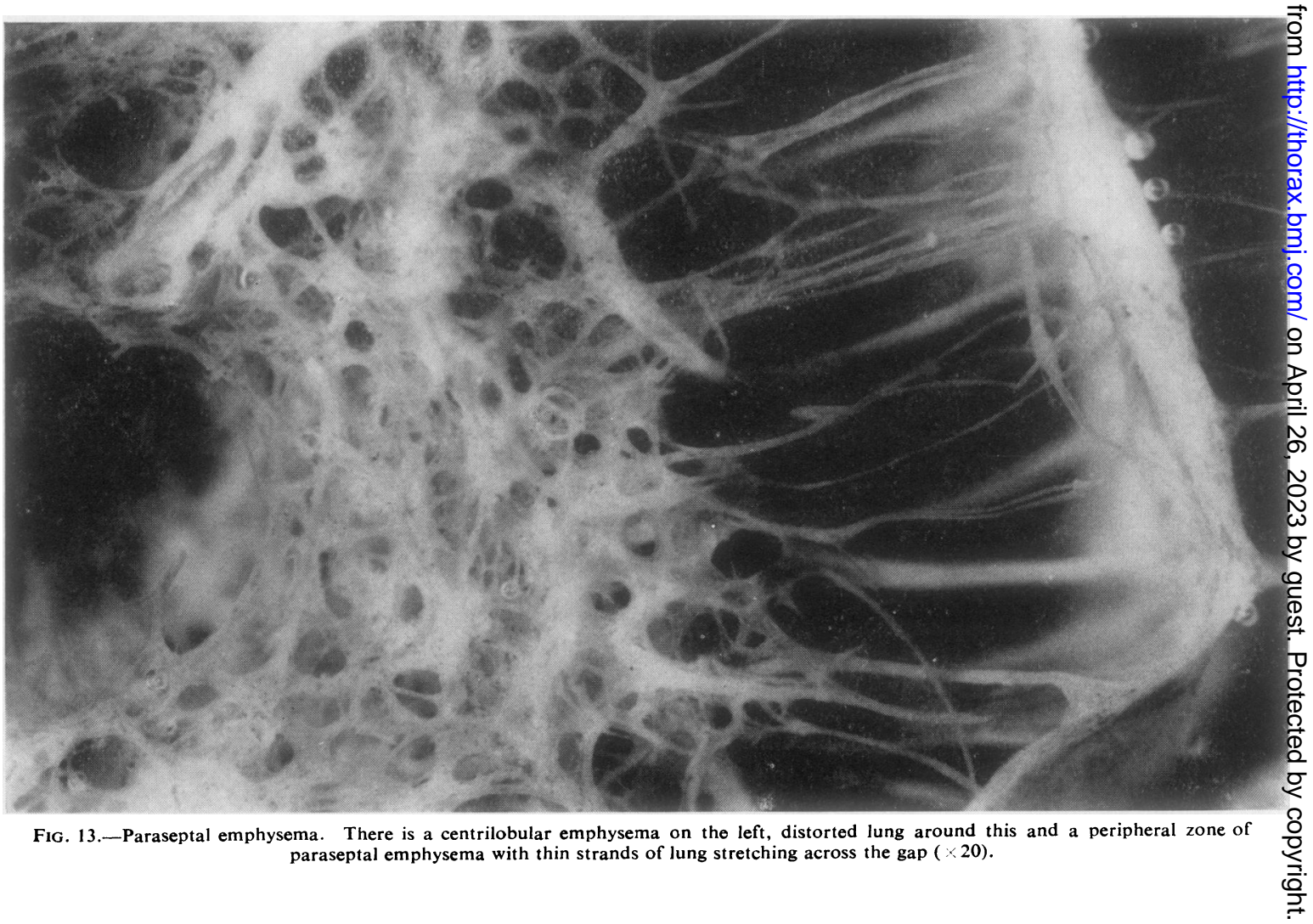


present results do not conflict with that, and although the present method is not so fine it will detect significant differences with age and with early diffuse emphysema. In fact, it is doubtful whether greater accuracy would give more useful information in view of the variability of alveolar contours.

No note has been made so far in this communication of the state of alveolar ducts, because it is not possible to identify them without serial sections. Heppleston (1953) thought that in "generalized" emphysema the respiratory bronchioles were little if at all enlarged, except perhaps for those of the third order. Alveolar ducts, atria, and alveolar sacs were the segments which characteristically showed an increase in diameter. It is difficult to compare our results, partly because of different methods which distend fine air spaces to different degrees, and partly because Heppleston's generalized emphysema may be different from the present diffuse emphysema (possibly one variety of it). All fine structures (beyond the terminal bronchioles) can be involved in diffuse emphysema and this applies both to dilatation in the mild form and to atrophy in the more severe forms.

McLean (1956) described nine cases of generalized diffuse emphysema and 28 where it was mixed with centrilobular emphysema. Later, McLean (1957b) traced a progression from centrilobular to diffuse emphysema as the periphery of the lobule was involved. Such a form has appeared in the present collection and is what I have termed severe centrilobular emphysema, that is, where confluent lesions involve a large proportion of the lung. I have kept this type classified as centrilobular emphysema even though all parts of lobules may be affected. In contrast to this, diffuse emphysema proper has no special distribution within the lobule, is only poorly pigmented, and is not necessarily accompanied by centrilobular emphysema. The differences are usually distinct in barium-impregnated slices. Only the occasional case is debatable.

While there are sharp differences between the centrilobular and diffuse forms of emphysema, it is necessary to emphasize strongly the fact that these types may be mixed in all proportions. The lung in Fig. 1e showed mostly diffuse emphysema and that in Fig. 11 mostly centrilobular emphysema. But each of these contained a trace of the other type and Fig. 9 shows moderate grades of both types mixed together. In the previous communication Figs. 11 and 12 showed widespread centrilobular emphysema with localized diffuse emphysema. Some chronic bronchitics have an intimate mixture of diffuse emphysema and a rather irregular type of centrilobular disease.

There is no single cause for all varieties of diffuse emphysema. The "vanishing lung" (Richards, 1956) is one which occurs in relatively young individuals, starting sometimes as early as the third decade and not associated with infection until far advanced. I think of this type as a developmental defect, but Richards has mentioned several other possibilities. Emphysema of childhood is in another group and often due to bronchial obstruction (see Jewett and Adler, 1958).

Sometimes diffuse emphysema seems to occur in one part of the lung as a compensation for shrinkage of another part (Fig. 9). The recent work of Leopold and Gough (1957) and McLean (1957a) indicates that centrilobular emphysema is due to recurring attacks of bronchiolitis. Several of my specimens of localized diffuse emphysema have had dense adhesions to the immediate pleura and are almost certainly inflammatory in origin. Some examples of centrilobular or diffuse emphysema have occurred near foci of tuberculous inflammation, a subject which Rappaport (1955) has discussed with reference to chemotherapy. The way in which diffuse emphysema is so often mixed with centrilobular emphysema, and the common occurrence of both these lesions in chronic bronchitics, also help to suggest that diffuse emphysema, like the centrilobular variety, may often be the end-result of acute inflammation. When diffuse emphysema is extensive, however, it is often difficult to decide whether infection was the cause or the effect. Careful clinicopathological studies will settle this problem.

\section{SUMMARY}

Methods for fixing lungs evenly and impregnating slices with barium sulphate can reveal the mildest grades of diffuse emphysema before histological examination.

Three normal lungs of different ages are described in detail and compared with examples of diffuse emphysema. In mild diffuse emphysema respiratory bronchioles and alveoli are dilated. In moderate diffuse emphysema there is loss of alveolar detail and a general thinning of air space walls. In severe cases there is loss of most fine air space walls.

Diffuse emphysema is contrasted with centrilobular emphysema. Although these are distinct 
forms they often occur in the same lung, sometimes intimately mixed.

Different varieties of diffuse emphysema are mentioned and their causation briefly discussed.

I am greatly indebted to Professor C. V. Harrison for his interest and advice. Mr. W. H. Brackenbury for his photographic work. Mr. J. G. Griffin for technical assistance, Miss V. Martin for secretarial assistance, and Mr. F. G. Saunders for drawing Fig. 1.
REFERENCES

Gough, J. (1957). Personal communication.

Hartroft, W. S., and Macklin, C. C. (1944). Trans, roy. Soc. C.ll. T (Sect. V, Biol Sci.), 3rd ser., 32, p. 63.

Heard, B. E. (1958). Thorax, 13, 136.

Heppleston, A. G. (1953). J. Path. Bact., C6, 235.

Jewett, T. C., and Adler, R. H. (1958). Surgery, 43, 926.

Leopold, J. G., and Gough, J. (1957). Thorax, 12, 219.

McLean, K. H. (1956). Aust. Ann. Med., 5, 73.

- (1957a). Ibid., 6, 124.

- (1957b). Ibid., 6, 203.

Rappaport, I. (1955). J. Amer. med. Ass., 158, 1436.

Richards. D. W. (1956). Bull. N.Y. Acad. Med., 32, 407. 